\title{
Morphological Development of the Gastrointestinal Tract Of Helmeted Guinea Fowl (Numida meleagris) at Pre-Hatch and Post-Hatch.
}

\section{I.J. Gosomji ${ }^{1}$, S.O. Salami ${ }^{2}$, J.O. Nzalak ${ }^{3}$, M.U. Kawu ${ }^{4}$, J.O. Omirinde ${ }^{1}$, N. Wanmi ${ }^{5}$ and P.D. Bukar ${ }^{6}$.}

${ }^{1}$ Department of Veterinary Anatomy, University of Jos, Plateau State, Nigeria.

${ }^{2}$ Department of Veterinary Anatomy, University of llorin, Kwara State, Nigeria.

${ }^{3}$ Department of Veterinary Anatomy, Ahmadu Bello University, Zaria, Kaduna State, Nigeria

${ }^{4}$ Department of Veterinary Physiology, Ahmadu Bello University, Zaria, Kaduna State, Nigeria.

${ }^{5}$ Department of Veterinary Anatomy, Federal University of Agriculture, Makurdi, Benue State Nigeria.

${ }^{6}$ Depot Nigerian Army/Nigerian Military School Medical Centre, Chindit Cantonment, Zaria, Kaduna State, Nigeria.

\section{Abstract}

This study was conducted to investigate the morphological development of the Helmeted Guinea fowl (Numida meleagris) gastrointestinal tract pre- and post-hatch. Eighty seven (87) eggs were purchased from the Poultry unit of National Veterinary Research Institute (NVRI), out of which eighty one (81) were used for pre-hatch and six (6) were allowed to hatch for post hatch studies. The development was recorded daily at pre-hatch while it was observed at day 1 and 8 at posthatch. The result revealed that at day 8 of incubation a digestive tube appeared with a roundish structure in the middle of the tube. By day 10

of incubation, a dilatation had appeared cranial to the roundish structure identified as the proventriculus. At the same time a small outgrowth appeared at the caudal end of the tube also identified as one of the caeca. By day 11 of incubation, the crop had appeared separating the oesophagus into cervical and thoracic parts, respectively. By days 12 and 13 of incubation the second caecum and the duodenal loop became apparent. With the appearance of the duodenal loop, the gross anatomical development of the guinea fowl GIT was completed. The residual yolk decreased with age until it became vestigial by day 8 post-hatch. This study revealed 
that days $8-13$ of incubation are the most critical period for the gross formation of the GIT in the guinea fowl.

Keywords: Development, GIT, incubation, Pre-hatch, Post-hatch.

\section{Introduction}

The development of embryonic gastrointestinal tract is characterized by vast structural and functional changes, which requires several processes such as induction and patterning of the endoderm and the recruitment of the mesoderm (Grappin-Botton and Melton, 2000; Stainier, 2002). This begins with the invagination of the cranial Intestinal Portal (AIP) and Caudal Intestinal Portal (CIP) at either end of the embryo that initiate the formation of the intraembryonic tube also called primitive gut lined by endoderm and covered by splanchnic mesoderm (Grappin-Botton and Melton, 2000; Poul et al., 2006). The primitive tube eventually became subdivided into three; foregut, midgut and hindgut (McGeady et al., 2006) with the foregut giving rise to the oesophagus, crop, proventriculus and ventriculus while the midgut forms the small intestine and the hind gut forms the large intestine (Drucilla et al., 1998).

Understanding the development of the gastrointestinal tract of helmeted

J. Vet. Anat. guinea fowl is critical in improving management and therapeutic approaches to maximize health and production efficiency (Sell et al,, 1991).

It is important to note that the plethora of works on guinea fowl focused on; studies on the onset of osteogenesis in grey-breasted helmeted guinea fowl (Salami, 2009), observations of the wattles of adult helmeted guinea fowl (Umosen et al., 2008), studies on the histochemistry of the proventriculus and gizzard of post-hatching guinea fowl (Senthanil et al., 2008), studies on the major respiratory pathways of the West African guinea fowl (lbe et al., 2008), Studies on the digestive, respiratory, urogenital system and lymphoid organs of helmeted guinea fowl (Lakshminarasimhan et al., 1983) and Studies on the external morphology and skeletal system (Ojo et al., 1983).

There is dearth of information on the gross morphogenesis of the gastrointestinal tract of helmeted guinea fowl during pre and post hatching. Therefore, this study was aimed at investigating the morphology of gastrointestinal tract of helmeted guinea fowl at both pre-hatch and posthatch and to determine the period during which the GIT is fully developed at pre-hatch in helmeted guinea fowl. 


\section{Materials and Methods}

\section{Egg Source and Preparation for Incubation.}

Eighty-Seven (87) fertilized guinea fowl eggs were purchased from National Veterinary Research Institute (NVRI), Vom, Plateau State, Nigeria. The eggs were incubated at Dhenab hatchery,Angul-D, Jos, Plateau State. Before incubation, the eggs were first cleaned with cotton wool wetted with tap water and allowed to dry at normal room temperature. They were then set in automatic electrical incubator (ASE EURO, Belgium); a twenty thousand $(20,000)$ capacity maintaining a temperature and relative humidity of $37.7^{\circ} \mathrm{C}$ and $60-70 \%$, respectively.

\section{Pre-hatch Study of the Embryos}

Starting from day one (1) of incubation, three (3) eggs were picked at random from the incubator. The removal of the embryos from the eggs was done according to the method described by Salami (2009). The shells of the eggs were cracked at the broad end with a forceps to create an opening of approximately one (1) inch in diameter. The outer and inner shell membranes were removed using a small pointed-end scissors. The cracked eggs were preserved in $10 \%$ formalin for 1 week to arrest further development of the embryo and harden the egg content for easier handling and ma- nipulation. The egg shells were removed and the embryos were exposed from under the shell membrane and placed on top of the yolk. With blunt forceps the embryos and the adherent extra-embryonic membranes were pulled away from the yolk and albumen. The extraembryonic membranes were removed and the umbilical stalks were severed close to the body wall. The harvested embryos were rinsed thoroughly under running tap water and then preserved in $10 \%$ formalin ready for use.

\section{Post-hatch Study of Keets}

Six (6) hatched keets out of the eighty-seven (87) fertilized eggs were used for the post-hatch study at day 1 and 8 post hatching. Three (3) keets were picked at random and sacrificed daily. The keets were euthanized with $0.1 \mathrm{ml}$ of phenobarbitone $200 \mathrm{mg} / \mathrm{ml}$ via jugular vein (Igwebuike and Eze, 2010).

\section{Morphological studies}

The gastrointestinal tracts of harvested embryos and keets were removed through the ventral incision into the thoraco-abdominal cavity. The gastrointestinal tract was carefully detached from the adhering structures and organs. Harvested gastrointestinal tract from the embryos and keets were carefully studied. Each segment of the gastroin- 
testinal tract was identified with the aid of magnifying lens. Photograph of all the segments of the gastrointestinal tract were taken as soon as they were identified.

\section{Results}

\section{Morphology at Pre-hatch and Post-hatch}

The guinea fowl embryo showed no clear morphological development of gastrointestinal tract from days 1-7 of incubation period. By day 8 of incubation, there appeared a straight tube with a roundish structure at almost the centre, thus presenting what later developed into the oesophagus, ventriculus and intestine craniocaudally (Fig 1A). These structures increased gradually in length and size till day 10 of incubation when a spindle-like structure proximal to the roundish ventriculus appeared as proventriculus and also a tiny out pocket lateral to the caudal straight tube appeared as one of the two caeca. Caudal to the caecum is a short tube representing colorectum (Fig 1B). At day 11 of incubation, an outpouch at the cranial part of the proximal tube appeared as the crop thereby dividing the oesophagus into the cervical oesophagus and thoracic oesophagus respectively (Fig 1C). At day 12 of incubation, the second caeca appeared at the opposite side of the first (Fig 1D). The duodenum which is the proximal part of the small intestine became apparent at day 13 of incubation. This part of the intestine became identifiable as a result of the tube having a U-shaped (Fig $2 \mathrm{E})$. It was also at this stage of development that the second caecum became conspicuous. Hence, the two caeca are now clearly seen at both sides of the intestine. The appearance of the caeca at day 13 of incubation completed the formation of the entire segments of the GIT and thus brings the developmental duration to six days in guinea fowl.

The gastrointestinal tract of guinea fowl at post-hatch showed no additional structural appearance except the increase in size, length, and weight of the formed structures. At post-hatch, the segments of the gastrointestinal tract took their normal position and location as found in the adult. The oesophagus of guinea fowl appeared at the right side of the neck. It appeared as cervical and thoracic oesophagus separated by an outpouch called crop (Fig 2F) a diverticulum of cervical oesophagus.

The stomach of guinea fowl has two portions; the glandular part known as proventriculus and the muscular part known as the ventriculus or gizzard. The proventriculus of guinea fowl appeared as a spindle shaped tube (Fig 2G), which began, 
distal to thoracic oesophagus at a junction called oesophagealproventricular junction and ends proximal to the muscular stomach (ventriculus) at a junction called isthmus gastris (Fig 2).

Ventriculus of guinea fowl is spherical in shape. It appeared at the left dorsal and ventral region of thoracoabdominal cavity and partly placed between and behind the lobes of the livers. The inner aspect of the ventriculus appeared to be lined by a hard structure called koilin or cuticila layer appearing partially green (Fig 2G).

The guinea fowl has a small intestine in the abdominal cavity. It is a longitudinal tube that continues distal to the pyloric end of ventriculus and ends proximal to the junction of caeca and colorectum (Fig 2G). The small intestine of guinea fowl like any other birds is divided into duodenum, jejunum and ileum. The duodenum appeared as a slightly elongated loop separated by pancreas into proximal descending and distal ascending parts (Fig 2G). The jejunum and ileum appeared long and coiled. They were separated by yolk sac that gradually reduced to become a rudimentary body called Meckel's diverticulum (Fig 2H).

Caeca of guinea fowl are elongated, paired blind sacs at both sides of the intestinal tube. The caeca is distal to the ileum and proximal to the colorectum. (Fig 2H).

The guinea fowl colorectum appeared as a short and straight tube extending from the distal part of the ileum and it opens distally into the the cloaca (Fig 2).

\section{Discussion}

The development of early digestive tract is very significant for achieving maximal growth in animals (Tako, 2004). It helps in determining the patterns of growth in the animal species, especially at different ages (Mobini, 2011) as it is possible to affect the biology and behaviour of the species (Sherri et al., 1988).

This study showed that oesophagus appeared as a short tube in guinea fowl at day 8 of incubation. This finding corroborates the vertebrates oesophageal development documented by (McGeady et al., 2006) which is reported to begins by extending as a fusiform dilation of the foregut and then separated into the cervical and thoracic oesophagus by the appearance of an out-pouch called the crop at day 11 of incubation. Also, similar observation was reported in chicken with early appearance of crop at day 8 of incubation (Daniel, 1957). The variation in the duration of crop appearance could be due to the incubation pe- 
riod which is 21 days in chicken and 28 days in the guinea fowl.

The avian stomach is peculiar in that it consists of two distinct physiological and morphological parts which are the glandular (proventriculus) and the muscular (gizzard) portions (Hodges, 1974; King and McLelland, 1984). The guinea fowl stomach was no exception to these distinct features. In addition to this, the isthmus was remarkably identified to appear as a constricted junction between the two portions of the stomach in the guinea fowl. This observation on isthmus agrees with earlier report in the pigeon by Hassan and Moussa (2012); While Hamida et al. (2013) observed that in Elanus caeruleus, the two portions of the stomach form one large pear-shaped cavity with no evidence of constricted junction (isthmus).

One of the striking observations in the development of the guinea fowl stomach was the embryonic ventriculus, which appeared two days earlier before the proventriculus. This observation revealed that the stomach development failed to follow the expected craniocaudal pattern that is peculiar to chicken. Though Briget (2006) reported the development of a swollen stomach at the $3 \frac{1}{2}$ days of incubation but there was no detailed information on the development of the portions of the stomach.

The segmentation of the intestine into small and large intestine became apparent with the appearance of one of the caeca at day 10 of incubation. The duodenal loop became apparent at day 13 of incubation making the distinction of the small intestine into the three segments of duodenum, jejunum and ileum possible. The transformation of the duodenal loop between day 1 and day 8 post-hatch was so marked. This observation con-curs with previous report of Nitsan et al. (1991) that emphasized the importance of digestive capacity for mucosal growth and function of the duodenum in the chicks during the early growth period. Also, similar study on goslings by Shih et al. (2005) documented closely related intestinal organ developmental patterns.

In this study, one of the two caeca appeared as a slight bulge at day 10 of incubation earlier than the second caecum at day 12 . This observation on the duration of caeca appearance contrasted the reports of Daniel (1957) and Helen et al. (2003) in the domestic fowl where the two caeca appeared at days 7 and 4 of incubation, respectively.

The colorectum in this study became distinguishable by day 10 of 
incubation in the guinea fowl when one of the two caeca appeared and served as the demarcation of the developing intestine into small and large intestine. Interestingly,in guinea fowl, the colon is not grossly distinguished from the rectum; But both had similar histological features of caecum. This finding is in line with what was reported for other galliform birds (Calhoun, 1954; Sell et al., 1991).

\section{Conclusion}

This study demonstrated that gastrointestinal tract growth and digestive functions are not fully developed in the newly hatched birds and that the GIT development does not follow the expected sequential cranio-caudal pattern in the guinea fowl. The development of the guinea fowl embryo takes 6 days to be completed; starting from day 8 of incubation with appearance of a straight tube having roundish structure at the centre and ending on day 13 of incubation with the appearance of both caeca.

\section{Acknowledgements}

To Mrs. Rahila Njam of Poultry section of the National Veterinary Research Institute, Vom, Plateau State, Nigeria for providing the required guinea fowl eggs for this research and also to Late Dr. Dege, for permitting me to use his incubator for this research.

\section{References}

Bridget, R.S (2006): Staging of intestinal development in the chick embryo. The Anatomical Record Part a 288a: 909-920.

Calhoun, M.L (1954): Microscopic Anatomy of the Digestive Systems of the Chicken. The lowa State Univ. Press, lowa.

Daniel, J.C (1957): An embryological comparison of the domestic fowl and the red-winged blackbird. Auk, 74: 340-358.

Drucilla, J.R; Devyn, M.S; Deborah, J.G and Clifford, J.T (1998): Epithelial-Mesenchymal signalling during the regionalization of the chick gut. Development, 125: 27912801.

Grapin-Botton, I and Melton, D.A (2000): Endoderm development: from patterning to organogenesis. Trends Genetics 16:124-130.

Hamida, H; Abdel-Wahab, E; Mostafa, $Z$ and Fathia, A (2013): Anatomical, histological and histochemical Adaptations of the avian alimentary canal to their food habits: II- Elanus caeruleus. International 
Journal of Scientific and Engineering Research , 4 (10): 1355 - 1364.

Hassan, S.A and Moussa, E.A (2012): Gross and Microscopic Studies on the Stomach of Domestic Duck (Anas platyrhynchos) and Domestic Pigeon (Columba livia domestica). Journal of Veterinary Anatomy, 5 (2): 105 - 127.

Helen, J.M; Bastian, F and Scott, E.F (2003): Wnt signaling components in the chicken intestinal tract. Developmental Biology, 256: 18-33.

Hodges, R.D (1974): The Histology of the Fowl. London: Academic Press.

Ibe, C.S; Onyeanusi, B.I; Salami, S.O; Umosen, A.D and Maidawa, S.M (2008): Studies of the major respiratory pathways of the West African Guinea fowl (Numida meleagris galeata): the morphometric and macroscopic aspect. International Journal of Poultry Science, 7 (10): $997-1000$

Igwebuike, U.M and Eze, U.U (2010) : Morphological characteristics of the small intestine of the African pied crow (corvus albus). Animal Research International, 7(1): 1116-1120.
King, A.S and McLelland, $J$ (1984): Birds: Their Structure and Function, 2nd edn. London: Bailliere Tindall.

Lakshminarasimhan, A; Ojo, S.A; Adogwa, A.O and Jamdar, M.N (1983): Studies on the anatomy of the grey breasted helmet guinea fowl (Numida meleagris galeata). Part 2: Digestive, respiratory, urogenital system and lymphoid organs. In: Helmeted guinea fowl, pp. 79-84.

McGeady, T.A; Quinn, P.J; FitzPatrick, E.S and Ryan, M.T (2006): Veterinary Embryology, pp. 209220.

Mobini, B (2011): Age - dependent morphometric changes of different parts of small and large intestine in the Ross broilers. International Journal of Agro Veterinary Science, 5 (5): 456-465.

Ojo, S.A; Lakshminarasimhan, A; Adogwa, A.O and Jamdar, M.N (1983): Studies on the anatomy of the grey breasted helmet guinea fowl (Numida meleagris galeata). Part 1: External morphology and skeletal system. In: Helmeted guinea fowl, pp. 73-77.

P. Hyttel; F. Sinowatz. and M. Vejlsted. (2010): Essentials of Do- 
mestic Animal Embryology. pp. 216. Saunders, Elsevier.

Nitsan, Z; Ben-Avzaham, G; Zoref, Z and Nir, I (1991): Growth and development of the digestive organs and some enzymes in broiler chicks after hatching. British Poultry Science, 32: 515-523.

Salami, S.O (2009): Studies on the onset of osteogenesis in greybreas-ted guinea fowl (Numida meleagris galeata). PhD. Thesis, Ahmadu Bello University, Zaria, Nigeria.

Sell, J.L; Angel, C.R; Piquer, F; Mallarino, J.E.G and Al-Batshan, H.A (1991): Developmental patterns of selected characteristics of the gastrointestinal tract of young turkeys. Poultry Science, 70: 12001205.

Senthanil, S.P; Ushakumary, S and Geetha, $\mathbf{R}$ (2008): Studies on the histochemistry of the proventriculus and gizzard of post-hatch guinea fowl (Numida meleagris). International Journal of Poultry Science, 7 (11): 111-116.
Sherri, L.M; Michael, A.G; Amy, R.K; Shawn, M.C; Mary, K.M; John, A.N; Barry, R.N and Richard, G.B (1988): Morphometric variation in tundra swans: relationships among sex and age classes. The Condor 90:802-815.

Shih, B.L; Yu, B and Hsu, J.C (2005): The development of gastrointestinal tract and pancreatic enzymes in White Roman Geese. Asian-Australian Journal of Animal Science 18 (6): 841-847.

Stainier, D.Y (2002): A glimpse into the molecular entrails of endoderm formation. Genes Development .16: 893-907.

Tako, E; Ferket, P.R and Uni, Z (2004): Effects of In ovo feeding of carbohydrates and $\beta$-hydroxy- $\beta$-methylbutyrate on the development of chicken intestine. Poultry Science, 83 : $2023-2028$.

Umosen, A.D; Onyeanusi, B.I; Salami, S.O; Nzalak, J.O; Imam, J and

\section{Corresponding author:}

Dr. Gosomji Innocent Jonah,

Department of Veterinary Anatomy, Faculty of Veterinary Medicine, University of Jos, Nigeria.

E-mail: innocentgosomji@yahoo.com 
A

$\infty$

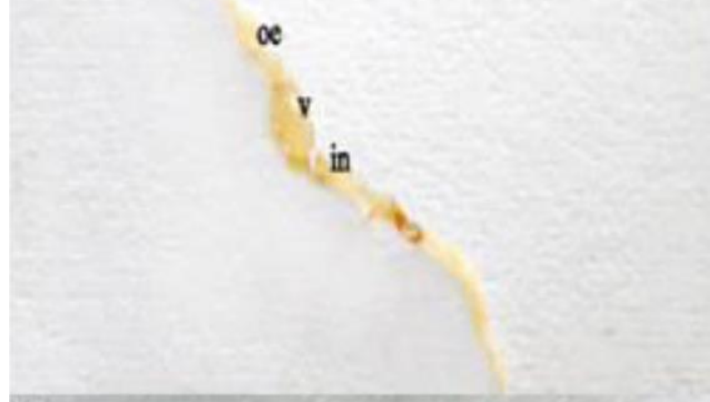

C



B

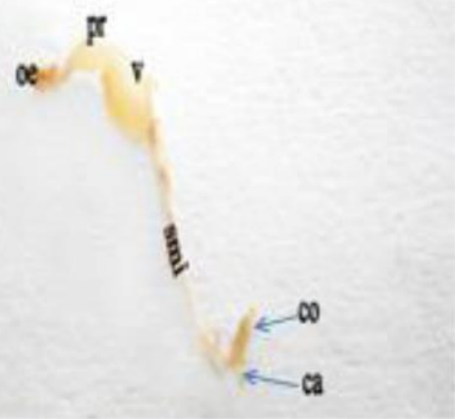

D

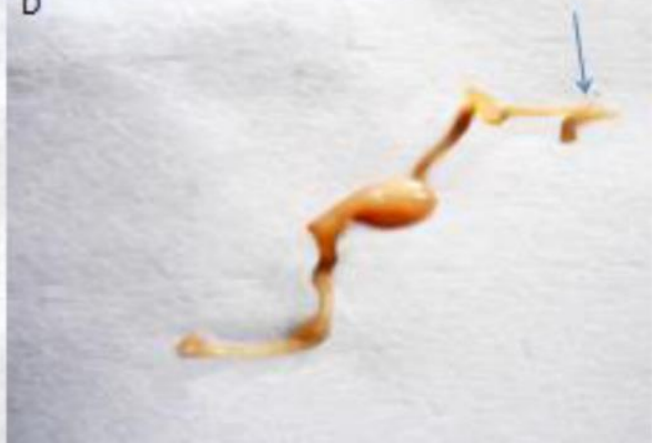

Fig (1): Gastrointestinal tract of guinea fowl embryo at day 8-12.

(A) Day 8 showing appearance of oesophagus (oe), ventriculus (v) and intestine (in);

(B) Day 10 showing oesophagus (oe), proventriculus (pr), ventriculus (v), small intestine (smi), caecum (ca) and colorectum (co);

(C) Day 11 crop (cr) appeared separating the oesophagus to cervical oesophagus (coe) and thoracic oesophagus (toe);

(D) Day 12 second caeca appearing as slight bulge (arrow). 


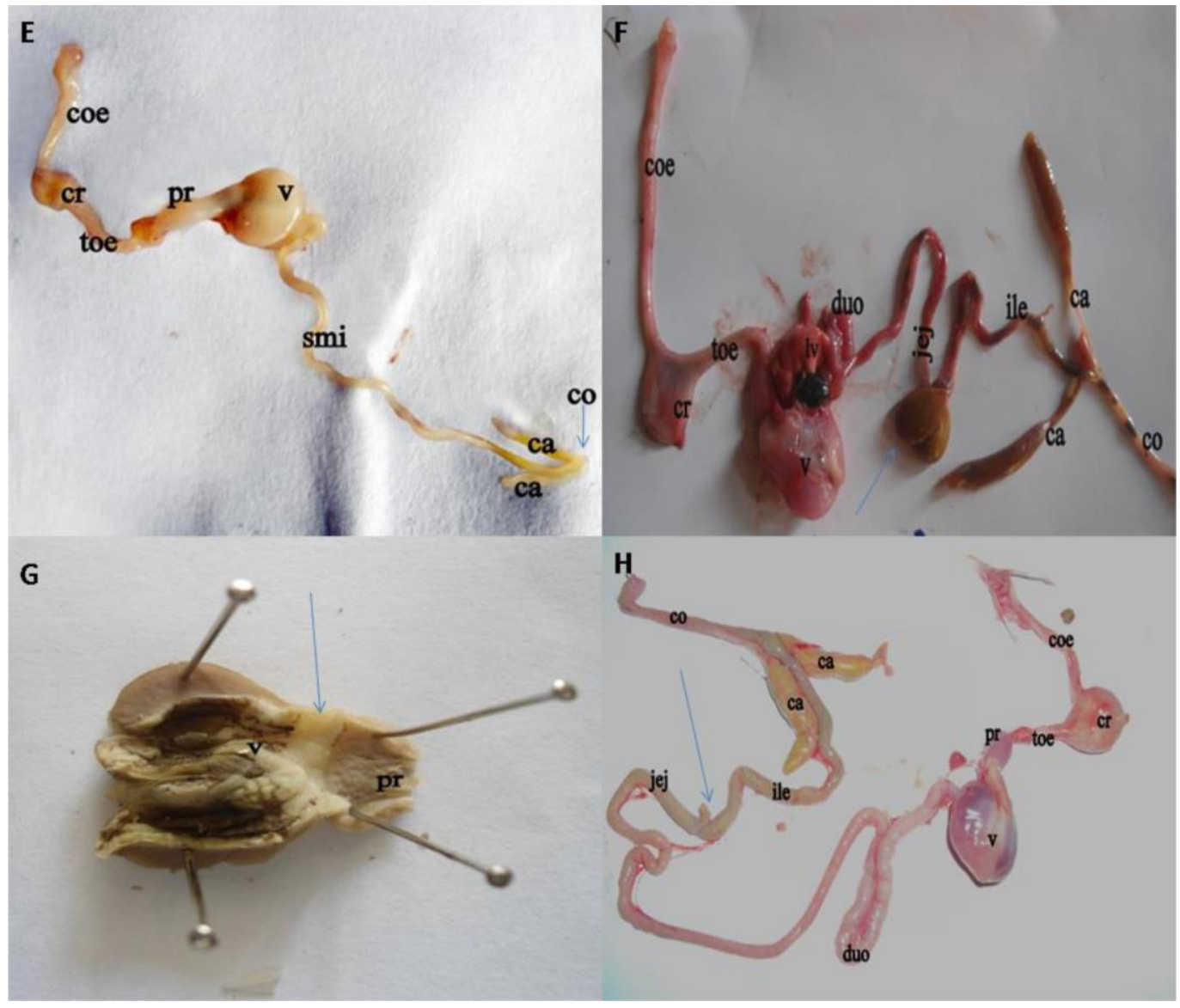

Fig (2): Gastrointestinal tract (GIT) of guinea fowl at pre-hatch and post-hatch.

(E) GIT of guinea fowl embryo at day 13 showing complete development of the segments with cervical oesophagus (coe), crop (cr), thoracic oesophagus (toe), proventriculus (pr), ventriculus (v), small intestine (smi), caeca (ca), colorectum (co) and the apparent duodenal loop (arrow);

(F) Day 1 post hatch showing complete segments with slightly large yolk (arrow);

(G) Cut open surfaces of proventriculus (pr) and ventriculus (v) separated by isthmus gastris (arrow);

(H) Day 8 post hatch showing complete morphological and functional GIT where the yolk sac has regressed to vetillinum diverticulum (arrow). 


\section{Helmeted Guinea fowl (Numida meleagris)}



Kingdom: Animalia \& Phylum: Chordata \& Class: Aves \& Order: Calliformes \& Family: Numidae \& Genus: Numide \& Species: N. meleagris

Helmeted, Grey-Breasted or Tufted Guineafowl: Frequently domesticated and included in exotic bird collections, the helmeted guineafowl is an easily recognizable bird and the most widespread game bird in Africa. In other parts of the world, feral and farm populations of these birds can be frequently seen and escapees are regular.

- Bill: Large, pale, upper mandible hooked, reddish base, whitish tip

- Size: 20-25 inches long with 38-inch wingspan, large round body, long neck, short tail, tiny head

- Colors: Blue, red, orange, black, white, brown, gray, buff

- Markings: Genders are similar with overall black or dark gray plumage with a dense, even pattern of white spots. On the wings, the spots are elongated to fine short bars. The neck is grayish black and may show a scruffy nape. The face is bare blue skin with bright red wattles at the base of the bill. The crown is topped with a horny brown or orange-brown "helmet" with a triangular horn shape. The thick legs and feet are gray.

Juveniles have similar markings but their wattles and horn are less developed and the overall colors are more grayish brown with whitish buff spots.

Source: http://birding.about.com/od/Grouse-Quail-And-Pheasants/p/HelmetedGuineafowl.htm (retrieved 19/7/2015) 